\title{
On the linkage between Gross Value Added by Economic Activities and the Overall Gross Value Added in EU-27
}

\author{
Francesca Dana ANDREESCU \\ Bucharest University of Economic Studies, Bucharest, Romania \\ andreescu.francesca@yahoo.com
}

\begin{abstract}
The financial and economic crisis led to a significant recession in the EU-27 in 2009, followed by a rebound in 2010. The existing economic situation requires a rethinking of the economic development policies, focused on analyzing the indicator of gross value added, created in production. This may lead to a new growth model, based on economic activities with higher value added. This paper investigates the linkage between overall gross value added in EU-27 and gross value added by economic activities, as described by NACE Rev. 2, from first quarter 2010 to second quarter 2020. The article attempts to include a wide range of statistical analysis and models for a complete assessment of the subject. Therefore, in order to achieve the objective, we choose to investigate the presence of causality relationships using VAR/SVAR models and Granger causality test, which reflect the presence of long and short-term relationships between certain selected variables. Through the assessment, we discovered a strong bi-directional causality between overall gross value added and the gross value added by industry and by distributive trades, transport, accommodation and food services, based on which we estimated a linear regression. The findings should present interest for policymakers, in order to assess perspectives to economic growth.
\end{abstract}

Keywords: EU-27 economic growth, gross value added, VAR/SVAR models, Granger causality, Regression.

\section{Introduction}

Dynamic interrelationships between different economic sectors have been commonly used in order to assess various shocks effect. The disaggregation of Gross Domestic Product (GDP) is based on demand side and supply side. The first concept refers to the fact that total production equals the total domestic demand and so the GDP represents the sum of public and private consumption, investments and net exports. The second concept which refers to production side of the economy states the fact that GDP consists of Gross Value Added (GVA) by sectors, import duties and Value Added Tax. Because the taxes and subsidies are usually very difficult to forecast, we concentrate the study on GVA.

Between 2010Q1 and 2020Q2, industry's share of EU-27 value added decreased 0.76 percentage points to $18.7 \%$, becoming the second largest activity after public administration, defense, education, human health and social work activities, which registered the largest increase during the period, up to $1.3 \%$, from 19.4\%. Even if wholesale and retail trade, transport, accommodation and food service activities decreased $2.26 \%$, it remains the third largest activity, followed by real estate activities and professional, scientific and technical activities, administrative and support service activities.

Gross value added can be used to provide essential quantitative economic details in order to establish or assess a policy intervention over a certain economic sector. Because some activities lead to higher GVA than others, it is important to identify the overall GVA variation cause and also the economic activity mix of a certain economy in order to evaluate the impact 
over productivity and economic growth. In other words, the GVA represents one of the most representative indicators in order to assess economic performance of a certain industry or sector.

\section{Literature review}

Researchers focus on the linkage between sectoral GVA distribution and structural changes. Pavelescu (2012) states the fact that sectoral structure of an economy is given also by the ratio of effective production to the value added and by backward multiplier, not only by the sectoral distribution of value added. The author presents the case of Romania during 1989-2009, highlighting the structural changes due to EU integration. He emphasizes the fact that in order to elaborate macroeconomic development policies it is important to take into consideration that a development of industry and construction activities may be a strong support for economic growth.

Robert Lehmann and Klaus Wohlrabe (2013) use an autoregressive distributed lag model in order to forecast the total and sectoral gross value added in the German economy at regional level, mainly because of the diversity of fiscal policies at sub-national level. They conclude the empirical method used to predict the sectoral GVA is more accurate than benchmark models, even if the accuracy differs significantly from one sector to another.

Nicu Marcu et al. (2014) states the fact that the correlation between sectoral GVA and GDP is important due to the information provided in regard with the factors influencing the GDP depending on the structure of GVA. The analysis is based on 2013 data and the authors also provide a regression equation, expressing GDP using as independent variables the structural elements of added value.

Vyklyuk Yaroslav and Valeriy Yevdokymenko (2016) provides evidence about how to determine future dynamics of economic activities with high value added. The authors use Hopfield`s neural network in order to assess the strategies of economic development.

Wlodzimierz Kolodziejczak (2020) analyzes the employment level and GVA per one person in agriculture by reference to other sectors of the EU economies.

All in all, the objectives of previous studies regarding GVA where to apply methods for estimating GVA at regional/ sub-national level or to highlight the linkages between certain activities gross value added and economic growth, in order to facilitate the development of sectoral growth policies with positive impact over GDP. Therefore, because of the EU integration and globalization process, we consider important to assess GVA as a suitable aggregate performance indicator due to the fact it does not introduce distortions between countries in different development stages. Moreover, in this way GVA allows to assess the economic and financial performance of EU-27 also by reflecting the structural differences.

\section{Methodology}

Analyzing the quarterly GDP between 2010Q1 and 2020Q2 from the output side gives an overview of the importance of 10 economic activities, as described by NACE Rev. 2, in terms of contribution to the overall gross value added at current prices. The assessment is performed based on Eurostat seasonally and calendar adjusted data. Estimates are made using quarterly data from 2010, first quarter, to second quarter of 2020. The data series used to assess the impact of the gross value added by main economic activities to the overall gross value added (OGVA) are: industry (I), wholesale and retail trade, transport, accommodation and food service activities, (RT) professional, scientific and technical activities, administrative and support service activities (PST), construction (Co) and information and communication (IC). In order to perform the assessment, we applied VAR, causality tests and SVAR. 
Based on VAR model, we propose to identify the relevant exogenous variables in order to assess the evolution of the overall gross value added at current prices. The first step is to establish the integration order of the variables by applying stationarity tests, followed by establishing the number of lags based on informational criteria, testing for cointegration, if needed, verifying if the model is stable, testing the residuals, identifying restrictions, if needed, and testing the coefficients stability.

VAR methodology is applicable only to same order stationary variables. As it is presented in literature, for quarterly data it is usually used a VAR model with 4 lags, thus implies an analysis based on maximum 6 variables. In this case, the exogenous selected variables are represented by the five main economic activities selected, while the endogenous variable is the overall gross value added. In order to set the optimal number of lags, there are used the information criterion Akaike, Hanna-Quinn, Schwartz tests and LR.

\section{Results and discussions}

The below table reflects the descriptive statistics of the selected variables in order to be analyzed. Series are positively skewed, most of them fairly symmetrical or moderately skewed, except the gross value added by construction, which is highly skewed with an asymmetry to the right, a kurtosis of 2.98, near the expected value, and the only variable not normally distributed, as shown by Jarque-Bera test. All other five variables series have the probability associated to Jarque-Bera test higher than 0.05 , and therefore they are normally distributed.

Table 1. Descriptive statistics for overall GVA and GVA by activity

\begin{tabular}{|c|c|c|c|c|c|c|}
\hline & OGVA & I & RT & PST & CO & IC \\
\hline Mean & 2753479. & 550941.6 & 523152.7 & 297096.1 & 146953.2 & 130960.4 \\
\hline Median & 2723997. & 548336.5 & 514834.5 & 292246.3 & 142003.1 & 126483.0 \\
\hline Maximum & 3153600. & 618757.7 & 607168.4 & 357739.8 & 177082.4 & 159332.8 \\
\hline Minimum & 2447377. & 481626.7 & 459177.9 & 247961.0 & 133147.4 & 113139.7 \\
\hline Std. Dev. & 213917.5 & 44076.91 & 45084.62 & 34135.12 & 12879.97 & 14668.86 \\
\hline Skewness & 0.431744 & 0.226848 & 0.426898 & 0.370186 & 1.112127 & 0.615678 \\
\hline Kurtosis & 1.840144 & 1.519683 & 1.795470 & 1.783131 & 2.982434 & 1.964037 \\
\hline Jarque-Bera & 3.571918 & 4.095179 & 3.723929 & 3.466073 & 8.452173 & 4.423651 \\
\hline Probability & 0.167636 & 0.129046 & 0.155367 & 0.176747 & 0.014609 & 0.109501 \\
\hline
\end{tabular}

Source: Authors`calculations.

The variables are tested for stationarity with the Augmented-Dickey-Fuller (ADF) test and the results are presented in Table 2. According to the ADF test, all six variables are integrated of order zero, therefore, by using VAR it can assess the causality between the selected variables.

Table 2. ADF test results

\begin{tabular}{|l|l|l|l|l|}
\hline \multirow{2}{*}{ Variabile } & \multirow{2}{*}{ Integration order } & \multicolumn{2}{c|}{ ADF results } & \multirow{2}{*}{ Exogenous } \\
\cline { 3 - 4 } & & \multicolumn{1}{|c|}{ t-statistic } & \multicolumn{1}{c|}{ p-value } & \\
\hline OGVA & $\mathrm{I}(0)$ & -5.329582 & 0.0000 & None \\
\hline $\mathrm{I}$ & $\mathrm{I}(0)$ & -2.016653 & 0.0431 & None \\
\hline RT & $\mathrm{I}(0)$ & -5.811197 & 0.0000 & None \\
\hline PST & $\mathrm{I}(0)$ & -3.335411 & 0.0197 & Constant \\
\hline Co & $\mathrm{I}(0)$ & -3.566674 & 0.0111 & Constant \\
\hline IC & $\mathrm{I}(0)$ & -3.220640 & 0.0262 & Constant \\
\hline
\end{tabular}


Table 3. Information criterion for choosing the number of lags

\begin{tabular}{|c|c|c|c|c|c|c|}
\hline Lag & LogL & LR & FPE & AIC & SC & HQ \\
\hline 0 & -2291.026 & NA & $1.29 \mathrm{e}+45$ & 120.8961 & 121.1547 & 120.9881 \\
\hline 1 & -1982.756 & 502.9658 & $7.89 \mathrm{e}+38$ & 106.5661 & $108.3761^{*}$ & 107.2101 \\
\hline 2 & -1937.122 & $60.04553^{*}$ & $5.51 \mathrm{e}+38^{*}$ & 106.0590 & 109.4204 & 107.2550 \\
\hline 3 & -1903.240 & 33.88174 & $9.36 \mathrm{e}+38$ & 106.1705 & 111.0833 & 107.9185 \\
\hline 4 & -1841.794 & 42.04200 & $6.55 \mathrm{e}+38$ & $104.8313^{*}$ & 111.2954 & $107.1312^{*}$ \\
\hline
\end{tabular}

Source: Authors`calculations.

PICBE | 1200

Based also on Akaike and Hannan-Quinn information criterions, the VAR(4) model is selected. The estimation is presented in Table 4, where for each lag, from 1 to 4, can be found the coefficients values together with the corresponding t-statistics.

Table 4. Estimation of VAR model

\begin{tabular}{|c|c|c|c|c|c|c|}
\hline & OGVA & I & RT & PST & $\mathrm{CO}$ & IC \\
\hline \multirow[t]{2}{*}{ OGVA(-1) } & -2.238 .193 & -1.036 .403 & -0.795206 & -0.494298 & -0.140346 & -0.086961 \\
\hline & {$[-0.89024]$} & {$[-1.48394]$} & {$[-0.87746]$} & {$[-1.24199]$} & {$[-0.79477]$} & {$[-0.84218]$} \\
\hline \multirow[t]{2}{*}{ OGVA(-2) } & -0.812289 & -1.102 .399 & -0.153634 & 0.327331 & -0.080488 & 0.105134 \\
\hline & {$[-0.21237]$} & {$[-1.03755]$} & {$[-0.11143]$} & {$[0.54063]$} & {$[-0.29961]$} & [0.66928] \\
\hline \multirow[t]{2}{*}{ OGVA $(-3)$} & 0.055478 & -0.209815 & 0.284873 & 0.075674 & -0.059925 & -0.036652 \\
\hline & {$[0.01565]$} & {$[-0.21305]$} & {$[0.22293]$} & {$[0.13485]$} & {$[-0.24066]$} & {$[-0.25173]$} \\
\hline \multirow[t]{2}{*}{ OGVA(-4) } & -2.116 .590 & 0.005289 & -0.906689 & -0.520999 & -0.097894 & -0.187758 \\
\hline & {$[-0.77613]$} & {$[0.00698]$} & {$[-0.92235]$} & {$[-1.20686]$} & {$[-0.51107]$} & {$[-1.67635]$} \\
\hline \multirow[t]{2}{*}{$\mathrm{I}(-1)$} & -0.126709 & 0.062546 & 0.013176 & 0.311264 & 0.110758 & 0.027521 \\
\hline & {$[-0.04251]$} & {$[0.07553]$} & {$[0.01226]$} & [0.65962] & [ 0.52899] & [ 0.22479] \\
\hline \multirow[t]{2}{*}{$\mathrm{I}(-2)$} & 0.778864 & 0.464301 & 0.333164 & -0.075382 & 0.148241 & -0.121062 \\
\hline & [ 0.21627$]$ & [ 0.46409$]$ & [ 0.25664$]$ & {$[-0.13223]$} & [ 0.58604$]$ & {$[-0.81847]$} \\
\hline \multirow[t]{2}{*}{$\mathrm{I}(-3)$} & -1.077 .187 & -0.194119 & -0.444437 & -0.266687 & 0.009100 & 0.009893 \\
\hline & {$[-0.30438]$} & {$[-0.19746]$} & {$[-0.34840]$} & {$[-0.47605]$} & {$[0.03661]$} & [0.06807] \\
\hline \multirow[t]{2}{*}{$\mathrm{I}(-4)$} & 3.799 .526 & -0.064723 & 1.599 .223 & 0.930400 & 0.272291 & 0.199619 \\
\hline & [ 1.22969] & {$[-0.07541]$} & [1.43588] & [1.90221] & [ 1.25467$]$ & [ 1.57304] \\
\hline \multirow[t]{2}{*}{ RT(-1) } & 1.219 .723 & 3.854 .322 & 4.272 .319 & 1.996 .178 & 0.435226 & 0.302086 \\
\hline & [2.55420] & [2.90550] & [2.48199] & [2.64068] & [ 1.29760] & [ 1.54026] \\
\hline \multirow[t]{2}{*}{ RT(-2) } & -4.730 .039 & 1.976 .334 & -2.471 .221 & -1.952 .312 & -0.604136 & -0.145330 \\
\hline & {$[-0.57913]$} & {$[0.87106]$} & {$[-0.83939]$} & {$[-1.51001]$} & {$[-1.05311]$} & {$[-0.43325]$} \\
\hline \multirow[t]{2}{*}{ RT(-3) } & 2.844 .093 & 1.822 .492 & 0.376588 & 0.080466 & 0.096400 & -0.078240 \\
\hline & [0.39695] & {$[0.91567]$} & {$[0.14581]$} & {$[0.07095]$} & {$[0.19156]$} & {$[-0.26588]$} \\
\hline \multirow[t]{2}{*}{ RT(-4) } & -1.966 .289 & 0.391557 & -0.827222 & -0.414356 & -0.357748 & 0.223904 \\
\hline & {$[-0.31178]$} & {$[0.22350]$} & {$[-0.36388]$} & {$[-0.41505]$} & {$[-0.80762]$} & [ 0.86444] \\
\hline \multirow[t]{2}{*}{ PST(-1) } & 6.779 .034 & 0.886556 & 2.329 .904 & 1.798 .404 & 0.797627 & 0.346542 \\
\hline & {$[0.93903]$} & {$[0.44208]$} & {$[0.89535]$} & {$[1.57370]$} & [ 1.57305] & [ 1.16879] \\
\hline \multirow[t]{2}{*}{ PST(-2) } & -1.413 .895 & 0.848687 & -0.856120 & -0.589229 & 0.067025 & -0.155627 \\
\hline & {$[-0.20200]$} & {$[0.43647]$} & {$[-0.33931]$} & {$[-0.53178]$} & [ 0.13633$]$ & {$[-0.54135]$} \\
\hline \multirow[t]{2}{*}{ PST(-3) } & 1.619 .550 & 1.149 .333 & 0.403661 & 0.135582 & 0.003615 & 0.090572 \\
\hline & {$[0.23803]$} & {$[0.60808]$} & {$[0.16459]$} & {$[0.12588]$} & {$[0.00756]$} & [0.32411] \\
\hline PST(-4) & 8.247 .615 & 1.586 .853 & 3.337 .973 & 1.068 .861 & 0.330445 & 0.451050 \\
\hline
\end{tabular}

DOI: 10.2478/picbe-2021-0111,pp. 1197-1207, ISSN 2558-9652 |

Proceedings of the $15^{\text {th }}$ International Conference on Business Excellence 2021 


\begin{tabular}{|c|c|c|c|c|c|c|}
\hline & OGVA & I & RT & PST & CO & IC \\
\hline & {$[1.32155]$} & {$[0.91532]$} & {$[1.48382]$} & {$[1.08193]$} & {$[0.75385]$} & {$[1.75975]$} \\
\hline $\mathrm{CO}(-1)$ & -0.109884 & 2.270 .200 & -0.157926 & -0.870885 & 0.276111 & -0.000199 \\
\hline & {$[-0.01502]$} & {$[1.11730]$} & {$[-0.05990]$} & {$[-0.75216]$} & {$[0.53745]$} & {$[-0.00066]$} \\
\hline $\mathrm{CO}(-2)$ & 4.497 .305 & 0.909689 & 1.850 .940 & 0.530036 & 0.664623 & -0.121682 \\
\hline & {$[0.68949]$} & {$[0.50205]$} & {$[0.78725]$} & {$[0.51334]$} & {$[1.45071]$} & {$[-0.45423]$} \\
\hline $\mathrm{CO}(-3)$ & -6.335 .851 & -2.671 .355 & -2.235 .693 & -0.582021 & -0.090010 & 0.067506 \\
\hline & {$[-0.95343]$} & {$[-1.44708]$} & {$[-0.93333]$} & {$[-0.55328]$} & {$[-0.19284]$} & {$[0.24734]$} \\
\hline $\mathrm{CO}(-4)$ & 0.197975 & -1.621 .842 & 0.637458 & 0.557683 & 0.060614 & 0.120754 \\
\hline & {$[0.02420]$} & {$[-0.71365]$} & {$[0.21617]$} & {$[0.43063]$} & {$[0.10549]$} & {$[0.35939]$} \\
\hline $\mathrm{IC}(-1)$ & 6.174 .028 & 2.912 .868 & 1.742 .958 & 0.665150 & 0.483796 & 0.410652 \\
\hline & {$[0.55683]$} & {$[0.94570]$} & {$[0.43610]$} & {$[0.37896]$} & {$[0.62123]$} & {$[0.90178]$} \\
\hline $\mathrm{IC}(-2)$ & 1.445 .366 & 2.544 .316 & 4.674 .217 & 2.321 .309 & 1.591 .696 & 0.107564 \\
\hline & {$[1.47846]$} & {$[0.93688]$} & {$[1.32643]$} & {$[1.49999]$} & {$[2.31805]$} & {$[0.26790]$} \\
\hline $\mathrm{IC}(-3)$ & -8.618 .452 & -2.211 .644 & -3.976 .771 & -0.835057 & 0.314113 & -0.161864 \\
\hline & {$[-0.90576]$} & {$[-0.83671]$} & {$[-1.15946]$} & {$[-0.55440]$} & {$[0.47000]$} & {$[-0.41419]$} \\
\hline $\mathrm{IC}(-4)$ & 6.520 .705 & -1.868 .224 & 2.178 .868 & 2.020 .994 & 0.217007 & 0.680876 \\
\hline & {$[0.55974]$} & {$[-0.57730]$} & {$[0.51888]$} & {$[1.09592]$} & {$[0.26521]$} & {$[1.42309]$} \\
\hline $\mathrm{C}$ & 3893441. & 1293609. & 1138550. & 427580.4 & 284226.1 & 113827.1 \\
\hline & {$[2.46795]$} & {$[2.95178]$} & {$[2.00215]$} & {$[1.71215]$} & {$[2.56506]$} & {$[1.75678]$} \\
\hline & & & & & Sourc: Authors`calations
\end{tabular}

PICBE | 1201

The graphic of the inverse roots of an autoregressive characteristic polynomial depicts the fact that the selected VAR model is not stable and, therefore, the process is explosive. Furthermore, one of the main hypotheses in the VAR methodology refers to the residual terms, which must be normal distributed, homoscedastic and with no autocorrelation.

Inverse Roots of AR Characteristic Polynomial

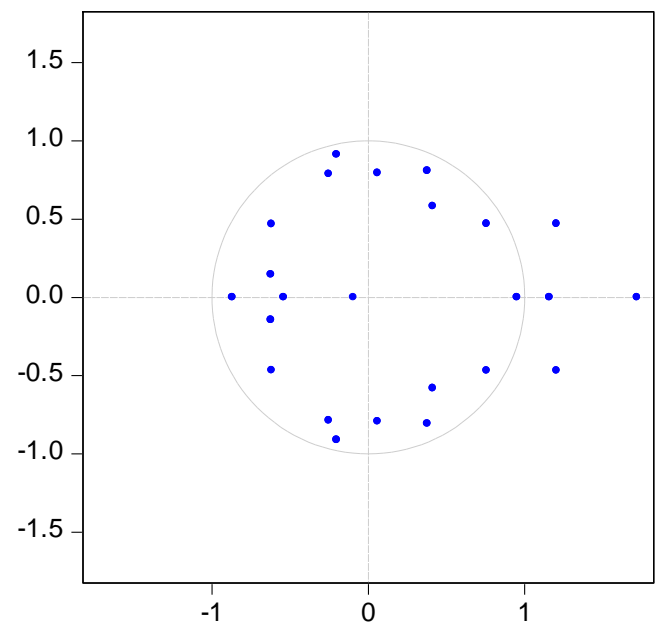

Figure 1. Inverse roots of AR characteristic polynomial

Source: Authors`calculations. 
Applying the Portmanteau test reflects the errors autocorrelation, while the p-value associated to Q-stat less than 0.05 leads to the rejection of the null hypothesis, meaning there is a contemporaneous influence in the model, which must be extracted.

Table 5. Portmanteau test results

\begin{tabular}{|c|c|c|c|c|c|}
\hline Lags & Q-Stat & Prob. & Adj Q-Stat & Prob. & df \\
\hline 1 & 47.72126 & NA* & 49.01102 & NA* $^{*}$ & NA* $^{*}$ \\
\hline 2 & 86.57900 & NA* & 90.02753 & NA* $^{*}$ & NA* $^{*}$ \\
\hline 3 & 121.6427 & NA* & 128.0967 & NA* $^{*}$ & NA* $^{*}$ \\
\hline 4 & 159.7316 & NA* & 170.6667 & NA $^{*}$ & NA* $^{*}$ \\
\hline 5 & 209.4526 & 0.0000 & 227.9211 & 0.0000 & 36 \\
\hline 6 & 238.6750 & 0.0000 & 262.6227 & 0.0000 & 72 \\
\hline 7 & 269.8356 & 0.0000 & 300.8196 & 0.0000 & 108 \\
\hline 8 & 314.1418 & 0.0000 & 356.9408 & 0.0000 & 144 \\
\hline 9 & 343.4807 & 0.0000 & 395.3849 & 0.0000 & 180 \\
\hline 10 & 363.8060 & 0.0000 & 422.9692 & 0.0000 & 216 \\
\hline 11 & 386.2288 & 0.0000 & 454.5272 & 0.0000 & 252 \\
\hline 12 & 420.3819 & 0.0000 & 504.4433 & 0.0000 & 288 \\
\hline
\end{tabular}

The residuals autocorrelation imposes re-estimating the VAR model either by using short or long term restrictions, either by combining the long term restrictions with the short term restrictions. The assessment of the overall gross added value by using a structural VAR (SVAR) is based on estimating the relation between the endogenous variable and the selected exogenous variables in order to determine permanent and temporary shocks. The advantage of this method consists in the possibility to obtain a better interpretation of the variations and results based on econometric considerations, without an arbitrary setting of the parameters values. To impose the restrictions is therefore used the Sims-Bernanke method, which facilitates reflecting the mutual causalities between variables. Depending on the relations between variables, the SVAR restrictions matrices $\mathrm{A}$ and $\mathrm{B}$ are defined, where $\mathrm{B}$ is a diagonal matrix and A describes the contemporaneous relations between variables. The restrictions are determined based on Granger causality test and taking into account theoretical considerations.

Table 6. The results of Granger causality test

\begin{tabular}{|l|l|l|r|r|}
\hline Lags: 4 & & & & \\
\hline Null Hypothesis: & F-Stat. & Prob. & Decision & Causality Type \\
\hline I does not Granger Cause OGVA & 5.19213 & 0.0028 & Reject H0 & Bi-directional Causality \\
\hline OGVA does not Granger Cause I & 19.7163 & $6 . \mathrm{E}-08$ & Reject H0 & Bi-directional Causality \\
\hline RT does not Granger Cause OGVA & 8.80550 & $9 . \mathrm{E}-05$ & Reject H0 & Uni-directional Causality \\
\hline OGVA does not Granger Cause RT & 1.52708 & 0.2204 & Do not reject H0 & Uni-directional Causality \\
\hline PST does not Granger Cause OGVA & 1.70723 & 0.1754 & Do not reject H0 & No Causality \\
\hline OGVA does not Granger Cause PST & 2.34148 & 0.0784 & Do not reject H0 & No Causality \\
\hline CO does not Granger Cause OGVA & 1.51155 & 0.2248 & Do not reject H0 & Uni-directional Causality \\
\hline OGVA does not Granger Cause CO & 7.22184 & 0.0004 & Reject H0 & Uni-directional Causality \\
\hline IC does not Granger Cause OGVA & 2.79026 & 0.0448 & Reject H0 & Bi-directional Causality \\
\hline OGVA does not Granger Cause IC & 8.22343 & 0.0001 & Reject H0 & Bi-directional Causality \\
\hline
\end{tabular}




\begin{tabular}{|l|r|r|r|r|}
\hline Lags: 4 & & & & Decision \\
\hline Null Hypothesis: & F-Stat. & Prob. & Causality Type \\
\hline RT does not Granger Cause I & 35.9440 & $8 . \mathrm{E}-11$ & Reject H0 & Bi-directional Causality \\
\hline I does not Granger Cause RT & 3.47256 & 0.0195 & Reject H0 & Bi-directional Causality \\
\hline PST does not Granger Cause I & 16.6485 & $3 . \mathrm{E}-07$ & Reject H0 & Bi-directional Causality \\
\hline I does not Granger Cause PST & 4.07647 & 0.0096 & Reject H0 & Bi-directional Causality \\
\hline CO does not Granger Cause I & 4.75318 & 0.0045 & Reject H0 & Uni-directional Causality \\
\hline I does not Granger Cause CO & 0.86864 & 0.4945 & Do not reject H0 & Uni-directional Causality \\
\hline IC does not Granger Cause I & 7.73522 & 0.0002 & Reject H0 & Bi-directional Causality \\
\hline I does not Granger Cause IC & 2.89699 & 0.0393 & Reject H0 & Bi-directional Causality \\
\hline PST does not Granger Cause RT & 0.31638 & 0.8647 & Do not reject H0 & Uni-directional Causality \\
\hline RT does not Granger Cause PST & 8.15984 & 0.0002 & Reject H0 & Uni-directional Causality \\
\hline CO does not Granger Cause RT & 1.24421 & 0.3143 & Do not reject H0 & Uni-directional Causality \\
\hline RT does not Granger Cause CO & 12.5277 & $5 . \mathrm{E}-06$ & Reject H0 & Uni-directional Causality \\
\hline IC does not Granger Cause RT & 1.62398 & 0.1949 & Do not reject H0 & Uni-directional Causality \\
\hline RT does not Granger Cause IC & 13.2563 & $3 . \mathrm{E}-06$ & Reject H0 & Uni-directional Causality \\
\hline CO does not Granger Cause PST & 1.57604 & 0.2072 & Do not reject H0 & Uni-directional Causality \\
\hline PST does not Granger Cause CO & 8.01312 & 0.0002 & Reject H0 & Uni-directional Causality \\
\hline IC does not Granger Cause PST & 1.75705 & 0.1646 & Do not reject H0 & Uni-directional Causality \\
\hline PST does not Granger Cause IC & 8.43968 & 0.0001 & Reject H0 & Uni-directional Causality \\
\hline IC does not Granger Cause CO & 2.02953 & 0.1164 & Do not reject H0 & Uni-directional Causality \\
\hline CO does not Granger Cause IC & 4.47824 & 0.0061 & Reject H0 & Uni-directional Causality \\
\hline & & & Source: Authors`calculations.
\end{tabular}

The interrelationships between the selected economic indicators are reflected in Table 6, using the results provided by Pairwise Granger causality test, which implies the rejection of null hypothesis at $\mathrm{p}$-value lower than 0.05 , where the null hypothesis is that first variable does not Granger cause the second variabile. Therefore, there is no causality between the gross value added by the professional, scientific and technical activities, administrative and support service activities and the overall gross value added. However, this indicator causes other activities gross value added with high impact over the total gross value added. There are bi-directional casualties, as for example, between gross value added by professional, scientific and technical activities, administrative and support service activities and gross value added by industry. Based on the Pairwise Granger causality test results, there can be established some linkages between indicators, also in line with the economic theory.

Table 7. Sims-Bernanke matrix decomposition for SVAR model

\begin{tabular}{|c|c|c|c|c|c|c|}
\hline & OGVA & I & RT & PST & CO & IC \\
\hline OGVA & 1 & NA(C4) & 0 & 0 & 0 & 0 \\
\hline I & NA (C1) & 1 & NA(C7) & 0 & NA(C12) & NA(C13) \\
\hline RT & 0 & NA(C5) & 1 & 0 & 0 & 0 \\
\hline PST & 0 & NA (C6) & NA(C8) & 1 & 0 & 0 \\
\hline CO & NA(C2) & 0 & NA(C9) & NA(C10) & 1 & 0 \\
\hline IC & NA(C3) & 0 & 0 & NA(C11) & 0 & 1 \\
\hline
\end{tabular}

DOI: 10.2478/picbe-2021-0111, pp. 1197-1207, ISSN 2558-9652 |

Proceedings of the $15^{\text {th }}$ International Conference on Business Excellence 2021 


\begin{tabular}{|c|c|c|c|c|c|c|}
\hline & OGVA & I & RT & PST & CO & 0 \\
\hline OGVA & NA(C14) & 0 & 0 & 0 & 0 & 0 \\
\hline I & 0 & NA(C15) & 0 & 0 & 0 & 0 \\
\hline RT & 0 & 0 & NA(C16) & 0 & 0 & 0 \\
\hline PST & 0 & 0 & 0 & NA(C17) & NA(C18) \\
\hline CO & 0 & 0 & 0 & 0 & 0 & NA(C19) \\
\hline IC & 0 & 0 & 0 & 0 & & 0 \\
\hline
\end{tabular}

PICBE | 1204

The results of the structural VAR analysis are represented by the impulse-response functions, which describe the innovation effects of one variable over the other variables, and by variance decomposition, which is useful to assess how shocks affect each economic variable in the system. In other terms, variance decomposition helps to determine the amount of the dependent variable variability explained by its own variance and which independent variable explains better over time the variability of the dependent variable. Impulse-response function together with variance decomposition permit to verify the behavior in the future of one variable as a response to the innovations of other variables.

Table 8. Variance decomposition

\begin{tabular}{|c|c|c|c|c|c|c|}
\hline Period & OGVA & I & RT & PST & CO & IC \\
\hline 1 & 100.0000 & 0.000000 & 0.000000 & 0.000000 & 0.000000 & 0.000000 \\
\hline 2 & 92.04778 & 2.427680 & 5.197792 & 0.251553 & 0.000169 & 0.075025 \\
\hline 3 & 88.24299 & 2.984006 & 8.430473 & 0.253399 & 0.007403 & 0.081728 \\
\hline 4 & 86.77543 & 3.047399 & 9.834964 & 0.241669 & 0.012701 & 0.087835 \\
\hline 5 & 86.22415 & 3.034192 & 10.39824 & 0.236364 & 0.015207 & 0.091838 \\
\hline 6 & 86.01923 & 3.020217 & 10.61590 & 0.234544 & 0.016301 & 0.093807 \\
\hline 7 & 85.94333 & 3.012783 & 10.69849 & 0.233966 & 0.016770 & 0.094661 \\
\hline 8 & 85.91520 & 3.009454 & 10.72958 & 0.233780 & 0.016968 & 0.095013 \\
\hline 9 & 85.90476 & 3.008072 & 10.74125 & 0.233717 & 0.017050 & 0.095155 \\
\hline 10 & 85.90087 & 3.007521 & 10.74562 & 0.233693 & 0.017084 & 0.095212 \\
\hline Period & OGVA & $\mathbf{I}$ & $\mathbf{R T}$ & $\mathbf{P S T}$ & $\mathbf{C O}$ & IC \\
\hline 1 & 71.28086 & 28.71914 & 0.000000 & 0.000000 & 0.000000 & 0.000000 \\
\hline 2 & 90.96366 & 2.274630 & 6.126434 & 0.524344 & $8.38 \mathrm{E}-05$ & 0.110847 \\
\hline 3 & 88.08400 & 2.485296 & 8.979332 & 0.350777 & 0.011104 & 0.089489 \\
\hline 4 & 86.69048 & 2.854601 & 10.07748 & 0.271693 & 0.015785 & 0.089958 \\
\hline 5 & 86.17807 & 2.970344 & 10.49695 & 0.244973 & 0.016843 & 0.092817 \\
\hline 6 & 85.99720 & 2.999880 & 10.65472 & 0.236830 & 0.017033 & 0.094338 \\
\hline 7 & 85.93362 & 3.006368 & 10.71350 & 0.234511 & 0.017071 & 0.094931 \\
\hline 8 & 85.91113 & 3.007434 & 10.73532 & 0.233886 & 0.017086 & 0.095139 \\
\hline 9 & 85.90311 & 3.007434 & 10.74343 & 0.233726 & 0.017096 & 0.095210 \\
\hline 10 & 85.90022 & 3.007319 & 10.74644 & 0.233687 & 0.017101 & 0.095235 \\
\hline
\end{tabular}

DOI: 10.2478/picbe-2021-0111, pp. 1197-1207, ISSN 2558-9652 |

Proceedings of the $15^{\text {th }}$ International Conference on Business Excellence 2021 


\begin{tabular}{|c|c|c|c|c|c|c|}
\hline Period & OGVA & I & RT & PST & $\mathrm{CO}$ & IC \\
\hline 1 & 97.07579 & 0.491511 & 2.432699 & 0.000000 & 0.000000 & 0.000000 \\
\hline 2 & 90.22609 & 2.581012 & 6.896771 & 0.223251 & $9.61 \mathrm{E}-05$ & 0.072781 \\
\hline 3 & 87.50492 & 2.995090 & 9.164991 & 0.245597 & 0.007038 & 0.082361 \\
\hline 4 & 86.49169 & 3.037118 & 10.12978 & 0.240328 & 0.012621 & 0.088455 \\
\hline 5 & 86.11767 & 3.026039 & 10.51248 & 0.236366 & 0.015263 & 0.092182 \\
\hline 6 & 85.97970 & 3.015929 & 10.65935 & 0.234680 & 0.016368 & 0.093977 \\
\hline 7 & 85.92869 & 3.010833 & 10.71488 & 0.234047 & 0.016813 & 0.094742 \\
\hline 8 & 85.90977 & 3.008632 & 10.73574 & 0.233815 & 0.016991 & 0.095051 \\
\hline 9 & 85.90274 & 3.007740 & 10.74355 & 0.233729 & 0.017062 & 0.095172 \\
\hline 10 & 85.90012 & 3.007390 & 10.74648 & 0.233697 & 0.017089 & 0.095219 \\
\hline Period & OGVA & I & RT & PST & $\mathrm{CO}$ & IC \\
\hline 1 & 92.30247 & 3.548916 & 0.107516 & 4.041097 & 0.000000 & 0.000000 \\
\hline 2 & 91.02045 & 3.088781 & 5.066180 & 0.773126 & 0.025166 & 0.026300 \\
\hline 3 & 88.11929 & 3.055519 & 8.406842 & 0.329442 & 0.019213 & 0.069690 \\
\hline 4 & 86.76592 & 3.043873 & 9.833669 & 0.252457 & 0.017032 & 0.087053 \\
\hline 5 & 86.22506 & 3.029271 & 10.39909 & 0.237304 & 0.016710 & 0.092561 \\
\hline 6 & 86.01972 & 3.018435 & 10.61645 & 0.234284 & 0.016816 & 0.094295 \\
\hline 7 & 85.94335 & 3.012343 & 10.69874 & 0.233738 & 0.016945 & 0.094882 \\
\hline 8 & 85.91513 & 3.009392 & 10.72968 & 0.233667 & 0.017027 & 0.095101 \\
\hline 9 & 85.90471 & 3.008083 & 10.74128 & 0.233668 & 0.017070 & 0.095188 \\
\hline 10 & 85.90085 & 3.007535 & 10.74563 & 0.233674 & 0.017091 & 0.095224 \\
\hline Period & OGVA & I & RT & PST & $\mathrm{CO}$ & IC \\
\hline 1 & 71.71787 & 1.253524 & 0.881024 & 0.604412 & 25.54317 & 0.000000 \\
\hline 2 & 87.38548 & 3.961915 & 3.131898 & 0.454641 & 5.018230 & 0.047836 \\
\hline 3 & 88.03619 & 3.606829 & 7.174578 & 0.432769 & 0.743346 & 0.006292 \\
\hline 4 & 87.01640 & 3.225175 & 9.297035 & 0.335888 & 0.091853 & 0.033646 \\
\hline 5 & 86.38136 & 3.072235 & 10.18791 & 0.277688 & 0.015156 & 0.065649 \\
\hline 6 & 86.09285 & 3.024265 & 10.53621 & 0.251324 & 0.012248 & 0.083101 \\
\hline 7 & 85.97420 & 3.011124 & 10.66875 & 0.240486 & 0.014816 & 0.090624 \\
\hline 8 & 85.92748 & 3.007926 & 10.71858 & 0.236237 & 0.016228 & 0.093554 \\
\hline 9 & 85.90949 & 3.007259 & 10.73720 & 0.234621 & 0.016792 & 0.094641 \\
\hline 10 & 85.90266 & 3.007158 & 10.74414 & 0.234019 & 0.016997 & 0.095033 \\
\hline Period & OGVA & I & RT & PST & $\mathrm{CO}$ & IC \\
\hline 1 & 50.56042 & 1.652960 & 6.697985 & 0.101122 & 2.339109 & 38.64841 \\
\hline 2 & 89.45216 & 3.327103 & 1.439976 & 0.612886 & 0.398815 & 4.769065 \\
\hline 3 & 89.99501 & 3.316067 & 5.770207 & 0.409310 & 0.044911 & 0.464496 \\
\hline 4 & 87.76786 & 3.159430 & 8.665010 & 0.296924 & 0.007241 & 0.103531 \\
\hline 5 & 86.64603 & 3.073019 & 9.931103 & 0.255924 & 0.009943 & 0.083985 \\
\hline
\end{tabular}

DOI: 10.2478/picbe-2021-0111, pp. 1197-1207, ISSN 2558-9652 |

Proceedings of the $15^{\text {th }}$ International Conference on Business Excellence 2021 


\begin{tabular}{|c|c|c|c|c|c|c|}
\hline 6 & 86.18506 & 3.034170 & 10.43609 & 0.241544 & 0.013836 & 0.089291 \\
\hline 7 & 86.00655 & 3.017930 & 10.63045 & 0.236486 & 0.015783 & 0.092804 \\
\hline 8 & 85.93899 & 3.011384 & 10.70403 & 0.234689 & 0.016594 & 0.094309 \\
\hline 9 & 85.91366 & 3.008803 & 10.73169 & 0.234045 & 0.016912 & 0.094894 \\
\hline 10 & 85.90420 & 3.007799 & 10.74205 & 0.233811 & 0.017033 & 0.095116 \\
\hline
\end{tabular}

Source: Authors`calculations.

The forecast horizon is in quarters. According to variance decomposition, the overall GVA variance is explained in proportion of $5.2 \%$ by the gross value added by wholesale and retail trade, transport, accommodation and food service activities in the second period and, in long-term, the proportion increase to almost $10.75 \%$. Also the gross value added by industry affects the overall GVA variance starting from $2.4 \%$ in the second period and it becomes stronger in the long-run. Even if the innovations registered at other activities gross value added level do not have a direct impact over. In the long-term the overall GVA contributes to over $85 \%$ of the industry GVA forecast error variance, and wholesale and retail trade, transport, accommodation and food service activities GVA contributes about $10.75 \%$. Construction GVA becomes stronger in the long-run, but is insignificant for explaining fluctuations in industry GVA evolution.

The gross value added by wholesale and retail trade, transport, accommodation and food service activities is highly affected by the overall gross value added, both in short- and long-run, and also by industry GVA, which contributes to RT variance with $3 \%$ on long-run to RT. Both fluctuations in GVA by professional, scientific and technical activities, administrative and support service activities and in gross value added by construction can be explained by the overall GVA, influence of which decrease slightly on long-run, and by RT GVA, which affects, for example, construction fluctuation with $3.13 \%$ on short-run and with $10.75 \%$ on long-run. Also variance of GVA by information and communication is stronger influenced by the overall GVA, industry GVA and by RT GVA.

As a preliminary conclusion, the SVAR analysis depicts the fact that overall GVA and GVA by economic activities are stronger influenced by the gross value added by most relevant activities in terms of proportion to the overall GVA. Therefore, we propose to construct a linear regression in order to express the overall GVA using two independent variables, selected based on SVAR analysis: industry GVA and wholesale and retail trade, transport, accommodation and food service activities GVA.

Table 9. Overall GVA regression

\begin{tabular}{|l|r|r|r|r|}
\hline \multicolumn{1}{|c|}{ Variable } & Coefficient & Std. Error & t-Statistic & Prob. \\
\hline \multicolumn{1}{|c|}{ I } & 2.624115 & 0.184124 & 14.25186 & 0.0000 \\
\hline RT(-1) & 2.498046 & 0.193804 & 12.88952 & 0.0000 \\
\hline R-squared & 0.990733 & Mean dependent var & 2753479. \\
\hline Adjusted R-squared & 0.990495 & S.D. dependent var & 213917.5 \\
\hline S.E. of regression & 20855.61 & Akaike info criterion & 22.77618 \\
\hline Sum squared resid & $1.70 \mathrm{E}+10$ & Schwarz criterion & 22.85977 \\
\hline Log likelihood & -464.9118 & Hannan-Quinn criter. & 22.80662 \\
\hline Durbin-Watson stat & 0.290576 & \multicolumn{2}{|l}{} \\
\hline
\end{tabular}

Source: Authors`calculations. 
The applied model is highly significant. The overall GVA is $99 \%$ explained by the current industry GVA and last period RT GVA. The coefficients are statistically significant at p-value of 0.01 . A variation of $1 \%$ in the current industry GVA leads to an increase of $2.62 \%$ in the overall GVA, while an increase of $1 \%$ in the last period RT GVA leads to an increase of $2.5 \%$ in EU-27 overall gross value added.

Conclusion

The aim of the study is to identify the existence of a significant relationship between overall GVA and the gross value added by each relevant type of activity in the EU-27 economy. Moreover, the study purpose is to identify the causality type describing the relationship between principal economic activities in terms of gross value added, using the five main economic activities in the EU-27 economy and the overall GVA. The result gives a general picture about the EU-27 economy in terms of impact of the main economic activities over the total GVA, suggested by the techniques used for the assessment: the structural VAR offers a clear quantitative overview, while the Granger causality test reaches to emphasise linkages which can be sustained by theoretical framework.

From a macroeconomic perspective, the assessment highlights the fact that, in the EU-27 economy, the development of wholesale and retail trade, transport, accommodation and food service activities and of activities in industry may be a great support for economic growth. Moreover, the overall gross value added is explained $99 \%$ by these variables between 2010 and second quarter of 2020.

\section{References}

Kolodziejczak, W. (2020). Employment and Gross Value Added in Agriculture Versus Other Sectors of the European Union Economy, Sustainability, 12(14), 5518.

Lehmann, R., Wohlrabe, K. (2013). Sectoral gross value-added forecasts at the regional level: Is there any information gain?, MPRA Paper 46765, University Library of Munich, Germany.

Marcu, N., Carstina, S. V., \& Marian, S. (2015). GDP correlation analysis with structural elements of added value, Procedia Economics and Finance, 22, 282-286.

Pavelescu, F. (2012). Fluctuation of economic activity, sectoral distribution of gross value added and the size of backward multipliers in Romania during the period 1989-2009, Romanian Journal of Economics, 35(2(44)), 88-112.

Vyklyuk, Y., Yevdokymenko, V., Yaskal, I. (2016). The Proportions and Rates of Economic Activities as a Factor of Gross Value Added Maximization in Transition Economy, Scientific Annals of Economics and Business, 63. 10.1515/saeb-2016-0104. 